The following resources related to this article are available online at www.sciencemag.org (this information is current as of October 14, 2009 ):

Updated information and services, including high-resolution figures, can be found in the online version of this article at:

http://www.sciencemag.org/cgi/content/full/326/5949/68

Supporting Online Material can be found at:

http://www.sciencemag.org/cgi/content/full/326/5949/68/DC1

A list of selected additional articles on the Science Web sites related to this article can be found at:

http://www.sciencemag.org/cgi/content/full/326/5949/68\#related-content

This article cites $\mathbf{4 4}$ articles, 9 of which can be accessed for free:

http://www.sciencemag.org/cgi/content/full/326/5949/68\#otherarticles

This article has been cited by 3 articles hosted by HighWire Press; see:

http://www.sciencemag.org/cgi/content/full/326/5949/68\#otherarticles

This article appears in the following subject collections:

Anthropology

http://www.sciencemag.org/cgi/collection/anthro

Information about obtaining reprints of this article or about obtaining permission to reproduce this article in whole or in part can be found at:

http://www.sciencemag.org/about/permissions.dtl 


\title{
The Ardipithecus ramidus Skull and Its Implications for Hominid Origins
}

\author{
Gen Suwa, Berhane Asfaw, Reiko T. Kono, Daisuke Kubo, C. Owen Lovejoy, Tim D. White
}

$\mathrm{T}$ he key feature that distinguishes Homo sapiens from other primates is our unusually large brain, which allows us to communicate, make tools, plan, and modify our environment. Understanding how and when our cognitive ability evolved has been a special focus in anthropology and, more recently, genetics. Fossil hominid skulls provide direct evidence of skull evolution and information about diet, appearance, and behavior. Skulls feature prominently in the characterization of species, in taxonomy, and in phylogenetic analyses of both extinct and living primates.

Unfortunately, hominid skulls are relatively rare in the fossil record. A number of partial skulls and crania (skulls without a lower

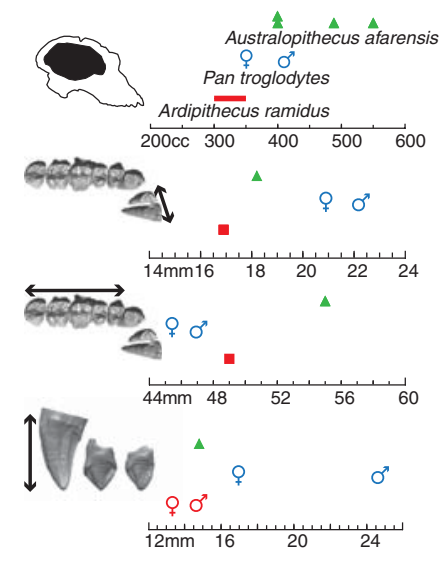

(Right) Oblique and side views of a female chimpanzee (right) and the $A r$. ramidus female reconstruction (left; the oblique view includes a separate mandible). (Left) Comparison of brain and tooth sizes (arrows) of chimps (Pan; blue), Ar. ramidus (red), and Australopithecus (green). Means are plotted except for individual Ar. ramidus and Au. afarensis cranial capacities. Canine unworn heights (bottom) are based on small samples, Ar. ramidus (females, $n$ $=1$; males, $n=3)$, Au. afarensis $(n=2)$, Pan (females, $n=19$; males, $n=11$ ). tus and are known as "robust" Australopithecus.

Ar. ramidus had a small brain ( 300 to $350 \mathrm{~cm}^{3}$ ), similar to that of bonobos and female chimpanzees and smaller than that of Australopithecus. The Ar. ramidus face is also small and lacks the large cheeks of "heavy chewing" Australopithecus. It has a projecting muzzle as in Sahelanthropus, which gives it a decidedly ape-like gestalt. Yet the Ar. ramidus skull is not particularly chimpanzee-like. For example, the ridge above the eye socket is unlike that of a chimpanzee, and its lower face does not project forward as much as a chimpanzee's face. Chimps primarily eat ripe fruits and have large incisors set in a projecting lower face. Ar. ramidus instead jaw) of early Homo and its predecessor, Australopithecus (which lived $\sim 1$ to 4 million years ago), have been recovered, but relatively few are complete enough for extensive comparisons. One surprisingly complete but distorted cranium from 6 to 7 million years ago was discovered in central Africa (Chad). This fossil, Sahelanthropus tchadensis (a.k.a. "Toumaï"), is thought by many to represent the earliest known hominid, although some have argued that it is a female ape.

The Ardipithecus ramidus skull is of particular interest because it predates known Australopithecus and thereby illuminates the early evolution of the hominid skull, brain, and face. The Ar. ramidus skull was badly crushed, and many of its bones were scattered over a wide area. Because the bones were so fragile and damaged, we imaged them with micro-computed tomography, making more than 5000 slices. We assembled the fragments into more than 60 key virtual pieces of the braincase, face, and teeth, enough to allow us to digitally reconstruct a largely complete cranium.

The fossil skulls of Australopithecus indicate that its brain was $\sim 400$ to $550 \mathrm{~cm}^{3}$ in size, slightly larger than the brains of modern apes of similar body size and about a third of those of typical Homo sapiens. Its specialized craniofacial architecture facilitated the production of strong chewing forces along the entire row of teeth located behind its canines. These postcanine teeth were enlarged and had thick enamel, consistent with a hard/tough and abrasive diet. Some species exhibited extreme manifestations of this specialized chewing appara- was probably more omnivorous and fed both in trees and on the ground. Additionally, in chimpanzees, forward placement of the entire lower face is exaggerated, perhaps linked with their large tusklike canines (especially in males) and elevated levels of aggression. This is not seen in Ar. ramidus, implying that it was less socially aggressive.

Like Ar. ramidus, $S$. tchadensis had a brain that was less than $400 \mathrm{~cm}^{3}$ in size. It also resembled Ar. ramidus in having small nonsharpened canines. Details of the bottom of the skull show that both Ar. ramidus and Sahelanthropus had a short cranial base, a feature also shared with Australopithecus. Furthermore, we infer that the rear of the Ar. ramidus skull was downturned like that suggested for Sahelanthropus. These similarities confirm that Sahelanthropus was indeed a hominid, not an extinct ape.

These and an additional feature of the skull hint that, despite its small size, the brain of Ar. ramidus may have already begun to develop some aspects of later hominid-like form and function. The steep orientation of the bone on which the brain stem rests suggests that the base of the Ar. ramidus brain might have been more flexed than in apes. In Australopithecus, a flexed cranial base occurs together with expansion of the posterior parietal cortex, a part of the modern human brain involved in aspects of visual and spatial perception.

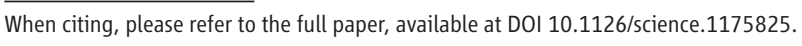




\section{The Ardipithecus ramidus Skull and Its Implications for Hominid Origins}

Gen Suwa, ${ }^{1 *}$ Berhane Asfaw, ${ }^{2}$ Reiko T. Kono, ${ }^{3}$ Daisuke Kubo, ${ }^{4}$ C. Owen Lovejoy, ${ }^{5}$ Tim D. White ${ }^{6}$

The highly fragmented and distorted skull of the adult skeleton ARA-VP-6/500 includes most of the dentition and preserves substantial parts of the face, vault, and base. Anatomical comparisons and micro-computed tomography-based analysis of this and other remains reveal pre-Australopithecus hominid craniofacial morphology and structure. The Ardipithecus ramidus skull exhibits a small endocranial capacity (300 to 350 cubic centimeters), small cranial size relative to body size, considerable midfacial projection, and a lack of modern African ape-like extreme lower facial prognathism. Its short posterior cranial base differs from that of both Pan troglodytes and $P$. paniscus. Ar. ramidus lacks the broad, anteriorly situated zygomaxillary facial skeleton developed in later Australopithecus. This combination of features is apparently shared by Sahelanthropus, showing that the Mio-Pliocene hominid cranium differed substantially from those of both extant apes and Australopithecus.

$\mathrm{T}$ he first fossil of Australopithecus, a partial child's skull found in 1924 at Taung, South Africa, was reported by R. A. Dart to combine an ape-like cranial capacity with distinctive hominid features such as weak facial prognathism, small anterior deciduous teeth, and an anteriorly situated foramen magnum (1). Since then, diverse Plio-Pleistocene cranial fossils have been recovered, primarily in southern and eastern Africa, establishing a widely recognized Australopithecus grade of evolution (2-6). Australopithecus crania exhibit small, chimpanzee-to-gorilla-sized cranial capacities, distinct cranial base flexion, and varying degrees of postcanine megadonty with associated craniofacial/vault morphologies $(2-5,7-10)$. The derivation of the genus Homo from Pliocene Australopithecus is probable (11), whereas the pre-Pliocene ancestry of Australopithecus has been elusive.

Until now, the only substantial specimen to shed any light on pre-Australopithecus hominid cranial evolution was that of Sahelanthropus tchadensis from Chad (12). Discovered in 2001, this Late Miocene cranium [specimen TM 26601-060-1; estimated at 6.0 to 7.0 million years ago (Ma)] combines a cranial capacity smaller than Australopithecus with a long and low neurocranium, an anteriorly extended upper face

${ }^{1}$ The University Museum, the University of Tokyo, Hongo, Bunkyo-ku, Tokyo, 113-0033, Japan. ${ }^{2}$ Rift Valley Research Service, Post Office Box 5717, Addis Ababa, Ethiopia. ${ }^{3}$ Department of Anthropology, National Museum of Nature and Science, Hyakunincho, Shinjuku-ku, Tokyo, 169-0073, Japan. ${ }^{4}$ Department of Biological Sciences, Graduate School of Science, the University of Tokyo, Tokyo, 113-0033, Japan ${ }^{5}$ Department of Anthropology, School of Biomedical Sciences, Kent State University, Kent, OH 44240-0001, USA ${ }^{6}$ Human Evolution Research Center and Department of Integrative Biology, 3101 Valley Life Sciences Building, University of California, Berkeley, CA 94720, USA.

*To whom correspondence should be addressed. E-mail: suwa@um.u-tokyo.ac.jp surmounted by a massive supraorbital torus with no post-toral sulcus, and a lower face less prognathic than those of either chimpanzees or gorillas (12-14). The posterior vault and cranial base are described as resembling post-3.5 Ma Pliocene Australopithecus (12-14). However, the hominid status of $S$. tchadensis has been challenged; some opined that it exhibits a surprisingly evolved face (15), whereas others have suggested it to be a gorilla ancestor or some other ape $(16,17)$.

We report here the skull of Ardipithecus ramidus recovered from Aramis, Ethiopia, as a part of the $A R A-V P-6 / 500$ skeleton (18). Together with other key Aramis specimens, including the $A R A-V P-1 / 500$ temporal/occipital portion (19), these fossils constitute the first substantial cranial remains of a pre-Australopithecus hominid directly associated with extensive postcranial remains (18). The Ar. ramidus postcranium indicates both substantial arboreal capability and an intermediate form of terrestrial bipedality that preceded the more fully established Australopithecus condition (20-23). The revelation of a primitive pre-Austalopithecus locomotor grade raises substantial interest in establishing the major features of the Ardipithecus cranium. Did Ar. ramidus share any of the derived hominid features seen in Australopithecus, or did it exhibit a skull more like those of extant African apes? What are its implications with respect to the controversies surrounding the hominid status of Sahelanthropus? We seek answers to these questions by comparing the Aramis fossils to Australopithecus, Sahelanthropus, and extant African apes, and we offer new hypotheses about cranial evolution in the hominid and African ape clades.

The ARA-VP-6/500 skull. The ARA-VP-6/ 500 skull comprises most of the vault, parts of the base, much of the right face, the left
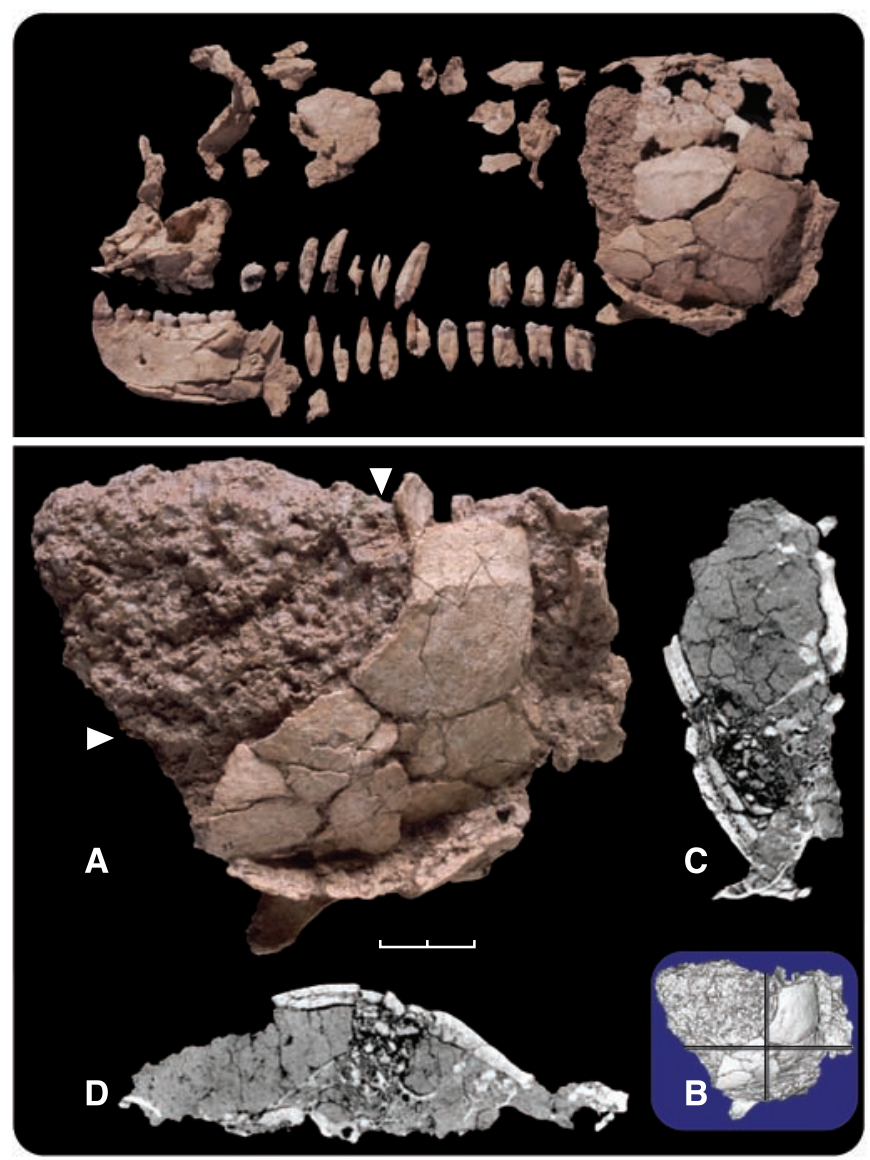

Fig. 1. The fragmented skull of $A R A-V P-6 / 500$. (Upper panel) Identifiable pieces of the skull after limited refitting for digital and physical molding. (Lower panel) (A) ARA-VP-6/500-032, (B) micro-CT rendered image of the same, with cross-sectional locations of (C) and (D) indicated. Arrowheads in (A) denote the positions of $(C)$ and (D). 
mandibular corpus, and most of the teeth (Fig. 1 and fig. S1). These elements were scattered widely across the excavation area (18). Many were partially disintegrated by the silty clay sediment, and major structures were fragmentary and variably distorted. Each was stabilized in the field, transported within its encasing sediment via plaster jacket, and later extracted from matrix under a binocular microscope.

The extracted pieces preserve contiguous bone from lambdoidal suture to face, but distortion prevented correct alignments (24). The largest intact element is most of the relatively well-preserved left parietal portion. It was collapsed into the vault space such that cranial height was reduced to $\sim 35 \mathrm{~mm}$ (Fig. 1). There has been excessive fragmentation and/or damage to the temporal and occipital portions. Individual pieces are so friable and soft that edge cleaning would have risked serious damage and loss of conjoint surface morphology. Therefore, major pieces were molded and otherwise left largely as recovered. Restoration was undertaken independently using casts (Berkeley, CA) and digital data (Tokyo, Japan).

In December 2003, we used high-resolution micro-computed tomography (CT) to scan the original fossils. We then segmented the representations of the better-preserved parts into 64 separate polygon shells. Using these digital models, we corrected the positions and alignments of each individual piece (24) (Fig. 2). We then added the digital model of the better-preserved $A R A-V P-1 / 500$ temporal/occipital fossil (19) (scaled to $92 \%$ size) to complete the $A R A-V P-6 /$ 500-based reconstruction of the Ar. ramidus cranium (25). The descriptions and comparisons that follow initially outline key features observed directly on the individually preserved fossils and then extend these to an analysis of the digital reconstruction.

Basion position and basicranial length. In our initial evaluation of Ar. ramidus (19), we noted the anterior position of the foramen magnum relative to lateral basicranial structures and interpreted this as a derived condition shared with later hominids. However, the utility of our observations has been questioned $(26,27)$. Here, we re-evaluate basion position and its importance in Ar. ramidus, using the newly available micro-CT data. These data allow high-resolution, three-dimensional wholesurface topographic assessment (Fig. 3). To minimize influences of orientation, we evaluated basion position in the basioccipital plane (Fig. 3) and confined our analysis to landmarks located inferiorly on the cranial base (i.e., excluding porion) (28). In $A R A-V P-1 / 500$, our digital methods yield a basion position $1.3 \mathrm{~mm}$ posterior to the center of the carotid foramen.

Previous workers have cautioned that because bonobos tend to have an anteriorly positioned foramen magnum (29), anterior placement of the basion might be primitive and therefore not a derived hominid feature (27). Thus, we com- pared basion position of Ar. ramidus with that of both Pan troglodytes and P. paniscus (24), as well as with Plio-Pleistocene Australopithecus. We found that although P. paniscus (mean $6.4 \mathrm{~mm}$, $n=28$ specimens) does have a slightly shorter basion-to-bicarotid distance than $P$. troglodytes (mean $7.3 \mathrm{~mm}, n=20$ ), this difference was not statistically significant. Furthermore, both species exhibit almost identical relative values when scaled by size (bicarotid breadth) (Fig. 3)

Basion position of Australopithecus overlaps minimally with the two Pan species (Fig. 3 and
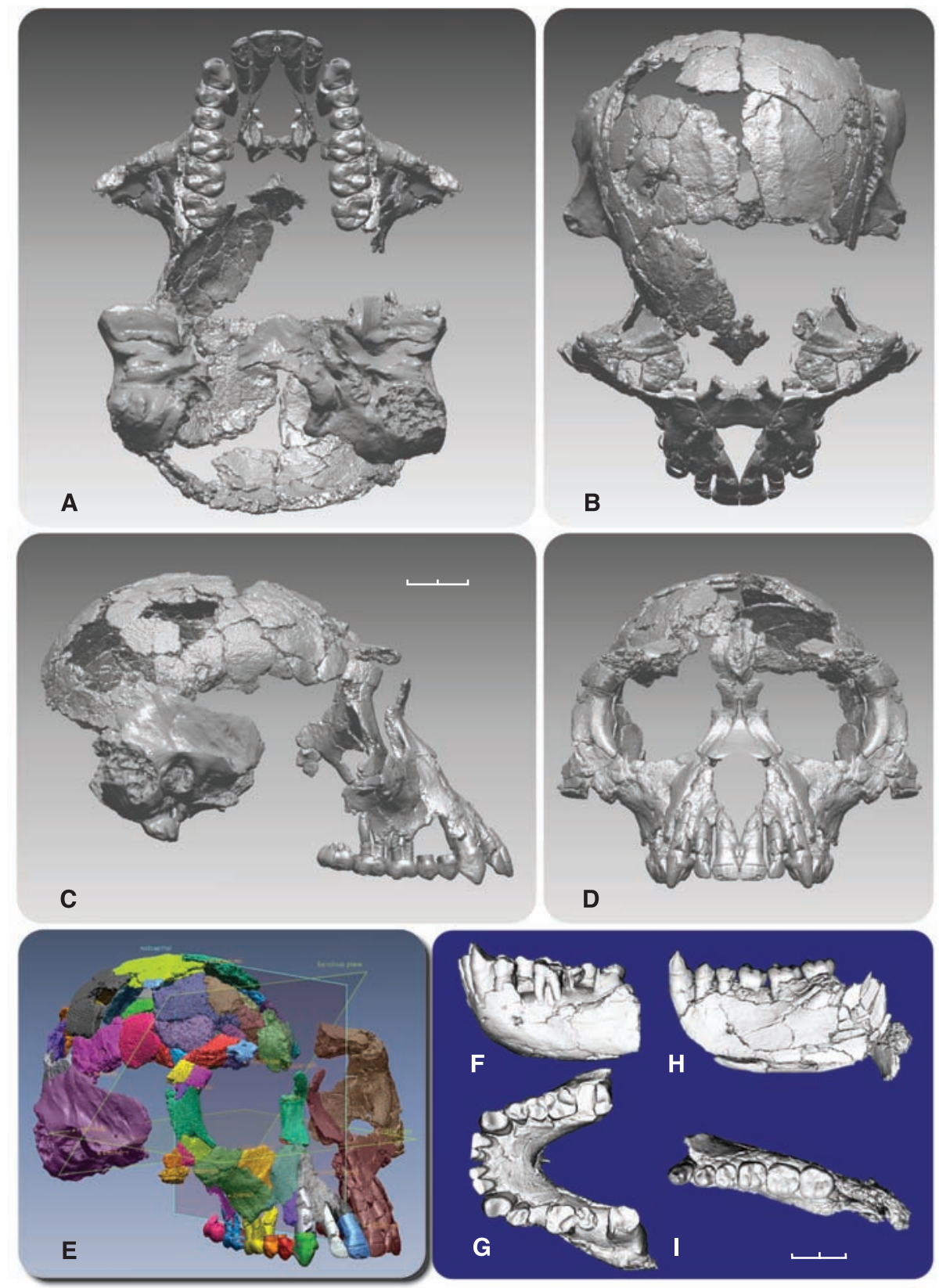

Fig. 2. Digital representations of the Ar. ramidus cranium and mandible. (A to D) The $A R A-V P-6 / 500$ and downscaled $A R A-V P-1 / 500$ composite reconstruction in inferior, superior, lateral, and anterior views (in Frankfurt horizontal orientation). (E) Individual pieces of the digital reconstruction in different colors. Note the steep clivus plane intersecting the cranial vault on the frontal squama (as in Sts 5 and not apes). (F and G) Lateral and superior views of the ARA-VP-1/401 mandible (cast). (H and I) Lateral and superior views of the $A R A-V P-6 / 500$ left mandibular corpus with dentition. 
slightly posterior to the bicarotid chord, as in the hominids examined here.

We also evaluated distance from the basion to the bi-foramen ovale (basi-ovale) chord as an alternative measure of posterior basicranial length (32) (figs. S3 and S4). Regression analysis in Pan shows a proportional relation between basi-ovale distance and basioccipital length. This relation also holds for Australopithecus. Therefore, basi-ovale distance can be used as a proxy for basioccipital length. Although $A R A-V P-1 / 500$ does not preserve the foramen ovale itself, the internal lateral wall of the foramen spinosum is preserved (Fig. 3), which permits a reasonable estimate of basi-ovale distance (24).

Ar. ramidus falls squarely within the hominid range (fig. S4). Although bonobos exhibit an absolutely shorter basi-ovale distance than do common chimpanzees throughout growth, this length relative to bicarotid breadth differs comparatively little between the corresponding age groups of the two species or among their growth periods (fig. S4). Thus, the wide and short posterior cranial base of $A r$ ramidus and Plio-Pleistocene hominids is not part of a continuum seen in modern ape morphology, but rather appears to reflect reorganization of the cranial base, most likely manifested early in ontogeny. Analysis of juvenile Australopithecus crania will allow a test of this prediction.

Though differences in posterior cranial base lengths and proportions are seen in the two Pan species, they show an even greater difference in their anterior cranial base lengths. Exocranially, this is reflected, for example, in metrics such as the distance from the foramen ovale to pterygopalatine fossa (fig. S5) and endocranially in the length of the planum sphenoideum (33). The morphological effects of these differences are a particularly elongate nasopharyngeal region with anterior placement of the palate and the entire dental arcade in P. troglodytes (fig. S6). Hence, the cranial base and facial hafting pattern of $P$. troglodytes appears highly derived relative to both $P$. paniscus and Ar. ramidus.

The $A r$. ramidus face and vault: basic morphology. The $A R A-V P-6 / 500-115$ maxilla exhibits a superoinferiorly short face and weak prognathism compared with the common chimpanzee. Its overall structure resembles that of Sahelanthropus, although it is smaller in size and proportionately shorter superoinferiorly. The preserved incisor alveoli and the size of its isolated roots/partial crowns indicates weak subnasal prognathism compared with both the common chimpanzee and the smaller-faced bonobo. This reflects the lack of incisor hypertrophy in Ar. ramidus (34). Facial topography from the infraorbital plane to the nasal aperture suggests that it had a short but projecting muzzle, considerably more primitive than the flatter-faced Plio-Pleistocene Australopithecus or the gracilized face of small Homo specimens such as $K N M-E R$ 1813. The zygomatic root of the maxilla (anterior face) is placed above the upper first molar $\left(\mathrm{M}^{1}\right)$, more posterior than is typical of Pliocene Australopithecus, but more anterior than is seen modally in bonobos and common chimpanzees. This reflects a less prognathic face compared with Pan and probably represents the primitive condition for both hominids and African apes. A similar zygomatic root location is found in many Miocene apes (e.g., Kenyapithecus, Nacholapithecus, Sivapithecus, Dryopithecus, Pierolapithecus, and Ouranopithecus).

The ARA-VP-6/500-115 maxilla exhibits a small but distinct upper second incisor/canine diastema (reportedly absent in Sahelanthropus and variable in $A u$. afarensis). Dental-arcade shape is observable in the ARA-VP-6/500 restoration and the $A R A-V P-1 / 401$ mandible (from an older presumed female) (Fig. 2). The mandible exhibits some primitive features, as well as some derived features shared with early Australopithecus. Although the canine-to-postcanine tooth row is straight in $A R A-V P-6 / 500$ [as it is in $A u$. anamensis (KNM-KP 29281) $(35,36)$ and some $A u$. afarensis (10)], the better-preserved $A R A-V P-1 / 401$ mandible exhibits an anteromedial position of the lower canine relative to lower third premolar, as in most $A u$. afarensis. However, the worn lower canine of $A R A-V P-1 / 401$ projects above both the postcanine occlusal and incisal planes, indicating that it was not incorporated into the functional incisive row, thus differing from Australopithecus (37).

Fig. 3. Basion position in ARA-VP-1/500. (A) Basal view (basioccipital plane horizontal). The two pieces were positioned by applying criteria of symmetry to the well-preserved basioccipital surface and by mirror imaging and determining overall best fit of the right and left sides (24). Metric landmarks (shown by red squares) are the basion, carotid foramen, and lateral margin of foramen spinosum (hidden). Two lines are drawn, depicting the sagittal plane (vertical line) and the bicarotid foramen chord (horizontal line). (B) Box plot of the basion-tobicarotid chord distance scaled by bicarotid breadth (24). The Australopithecus specimens measured
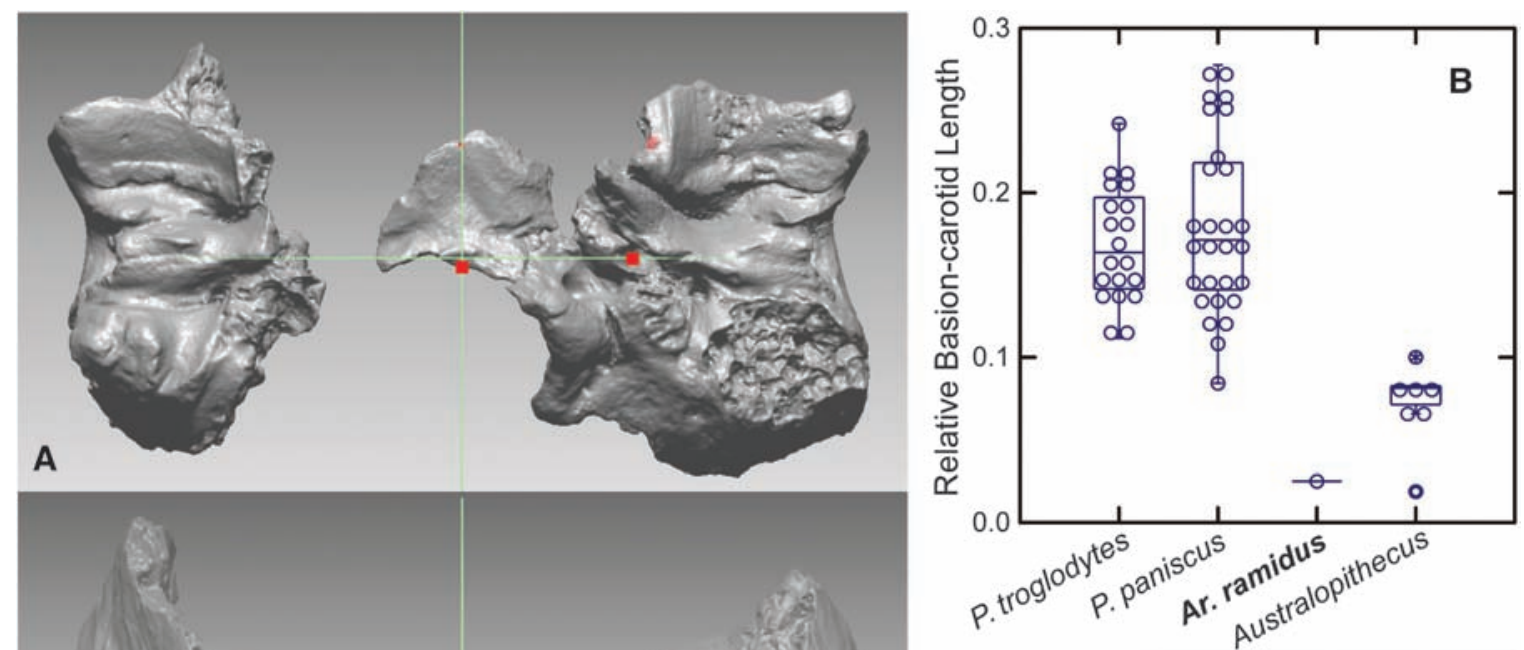

were as follows: Sts 5, Sts 19, MLD $37 / 38$ (casts of Au. africanus); KNM-WT 17000 (Au. aethiopicus); and O.H. 5 (cast), KNM-ER 406, KNM-ER 407 (Au. boisei) (see fig. S2 for individual values). (C) Anterior view showing segmented internal ear. The validity of the bilateral placements was evaluated by examining semicircular canal asymmetry, which was confirmed to be slight and within ranges observed in humans $(57,58)$. Radii of the semicircular canals were measured as in $(59)$ and were found comparable to the modern ape and Australopithecus conditions (60).
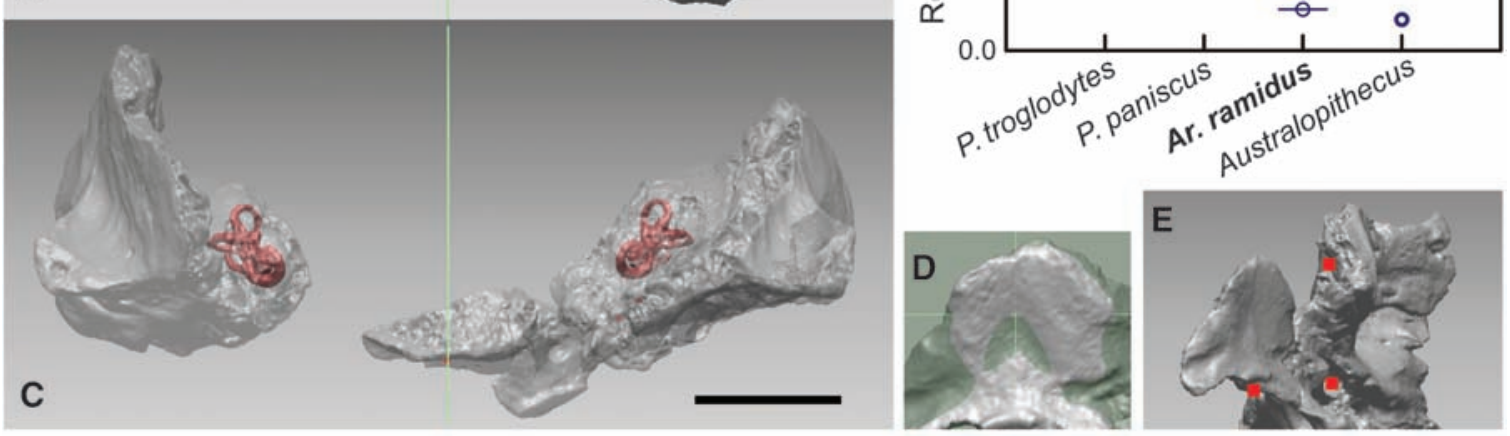

(D) Close-up of basioccipital showing a horizontal plane passing through the midbasioccpital point (24). Note the approximate symmetry of the basioccipital surface [1.5 times the scale of (A), (C), and (E)]. (E) Oblique basal view showing the three landmarks (the basion, carotid foramen, and lateral margin of foramen spinosum). The latter was used in alternative measures of cranial base length (24) (see text and figs. S3 and S4 for further details and discussion). Scale bar, $20 \mathrm{~mm}$; common to $(A),(C)$, and $(E)$. 
Fig. 4. Major structural features (24) of the Ar. ramidus cranium (ARA-VP-6/500-based reconstruction). (A) Upper facial projection: ratio of porion-to-nasion radius by porionto-prosthion radius. S. tchadensis (14); Australopithecus includes Sts 5 (this study), Sts 71 (9), and O.H. 5 (14); KNMER 1813 from CT scan of cast (this study). The Ar. ramidus value is 0.73 . (B) Midfacial projection: ratio of porion-tonasal aperture radius by average of porion-to-orbitale and porion-to-zygomatic root radii. Australopithecus is Sts 5 (this study); KNM-ER 1813 from CT scan of cast (this study). The Ar. ramidus value is 1.23. (C) Facial mask index: maximum zygomatic breadth at orbital plane divided by biorbital breadth across ectoconchion. S. tchadensis (14); Australopithecus includes A.L. 444-2, Sts 5, Sts 71, SK 48, TM 1517, O.H. 5, KNM-ER 406, KNM-ER 732, KNM-ER 13750, and KNM-WT 17000 (10). The Ar. ramidus value is 1.20. (D) Overlap index: ratio of projected glenoid tubercle-toprosthion length to projected zygomaxillare-to-distal $\mathrm{M}^{3}$ distance. Australopithecus includes A.L. 444-2, Sts 5, Sts 71, SK 48, SK 52, TM 1517, O.H. 5, KNM-ER 406, KNM-ER 732, and KNM-WT 17000 (10). The Ar. ramidus value is 0.26 . (E) Subnasal alveolar prognathism: ratio of porion-to-prosthion radius by porion-to-nasal aperture radius. Australopithecus is Sts 5 (this study); KNM-ER 1813 from CT scan of cast (this study). The Ar. ramidus value is 1.23 . (F) Relative upper facial breadth: bi-frontomalare temporale breadth divided by cube root of cranial capacity. S. tchadensis (13); Australopithecus is divided into nonrobusts (A.L. 444-2, Sts 5, Sts 71, and Stw 505) and robusts (O.H.5, KNM-ER 13750, and KNM-ER 23000); data for these and KNM-ER 1813 compiled from $(9,10,47,61)$. The Ar. ramidus value is 15.4 . (G) Relative palatal length: projected palate (or dental row) length divided by cube root of cranial capacity. Australopithecus is divided into nonrobusts (A.L. 444-2, Sts 5, and Sts 71) and robusts (O.H. 5 and KNM-WT 17000); data for these and KNM-ER 1813 compiled from (10). The Ar. ramidus value is 9.1. (H) Relative bi-glenoid breadth: biexternal glenoid tubercle breadth divided by cube root of cranial capacity. S. tchadensis [estimated from (13)]; Australopithecus divided into nonrobusts (A.L. 444-2, Sts 5, and MLD 37/38) and robusts (O.H. 5, KNM-ER 13750, KNMER 23000, and KNM-WT 17000); data for these and KNM-ER 1813 compiled from (10). The Ar. ramidus value is 15.8. See SOM materials and methods (24) for further details.
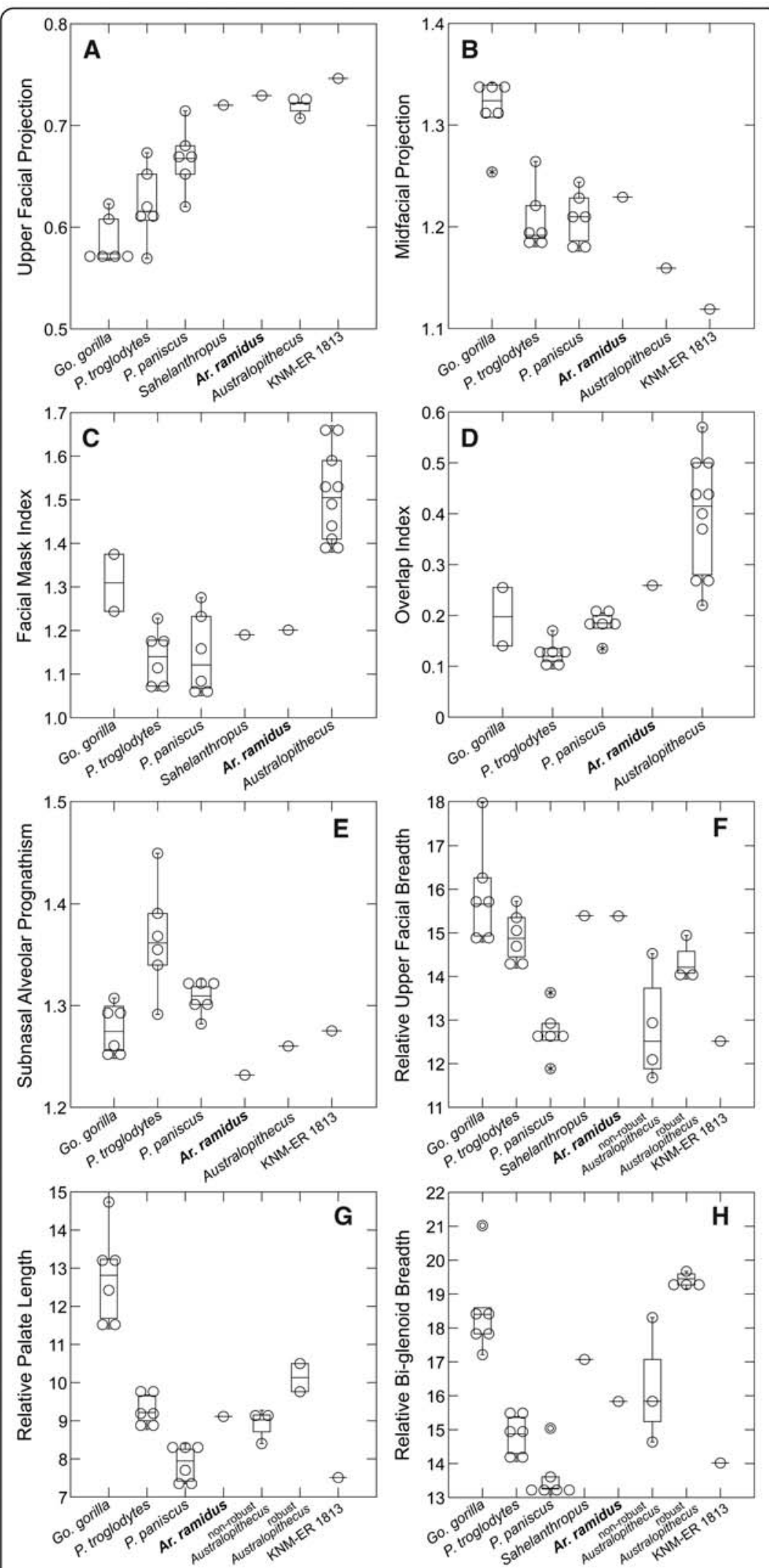
In mandibular corpus morphology, $A R A-V P$ 1/401 exhibits a posteriorly receding symphysis and lateral corpus proportions resembling $A u$. anamensis rather than Pan. However, compared with $A u$. anamensis, both $A R A-V P-1 / 401$ and $A R A-V P-6 / 500$ exhibit a less inflated mandibular corpus, accompanied by extensive lateral hollowing and high posterior placement of the ramus root. The Ar. ramidus mandible is similar to those of Sahelanthropus and Ar. kadabba in corpus dimensions $(12,38,39)$, ramus root position and development, and circum mid-corpus height placement of the anterosuperiorly exiting mental foramen.

The Ar. ramidus supraorbital torus is represented by a small but informative segment of the $A R A-V P-6 / 500$ frontal bone. The torus is vertically $6 \mathrm{~mm}$ thick at about mid-orbital position, a location commonly thinnest in African apes. It is equivalent to the thinnest end of the $P$. troglodytes range (40). Although there is considerable individual variation and overlap of ranges in torus thickness between the sexes of modern apes $(12,17)$, the thin supraorbital torus of $A R A-V P$ $6 / 500$ suggests that this individual was female, supporting our sex assignment on the basis of canine size (34). Concavity behind the torus is slight, indicating the absence of Gorilla- or Pan-like post-toral sulci. The lateral wall of the frontal sinus is exposed on the medial break of the preserved supratoral region. Thus, the presence of a sizeable frontal sinus is shared with both Pan and Gorilla.

The frontozygomatic region lateral to the orbit of the Ar. ramidus cranium is wide and rugose, comparable to robust individuals of P. troglodytes, and distinctively more robust than in the bonobo. The temporal line turns posteriorly at about mid-orbital position, comparable to Sahelanthropus, and well within the wide $P$. troglodytes range of variation. The superior temporal line then runs largely anteroposteriorly for the length of the parietal portion (right and left lines separated by $\sim 25 \mathrm{~mm}$ ) and crosses the lambdoidal suture. The crushed occipital region does not allow for comprehensive evaluation of compound temporal/nuchal crest configuration, but a small compound crest is preserved laterally on the left side. Such a crest is variably expressed in both male and female P. troglodytes but is typically absent in bonobos (both sexes). A similar crest is commonly seen in Au. afarensis (10).

In summary, the facial bones of $A R A-V P-6 /$ 500 suggest that prognathism is weaker than in Pan, but that the masticatory complex is more developed than in bonobos, consistent with the larger Ar. ramidus postcanine dentition (34). The $A R A-V P-6 / 500$ face is markedly short superoinferiorly, but modern ape data $(10,41,42)$

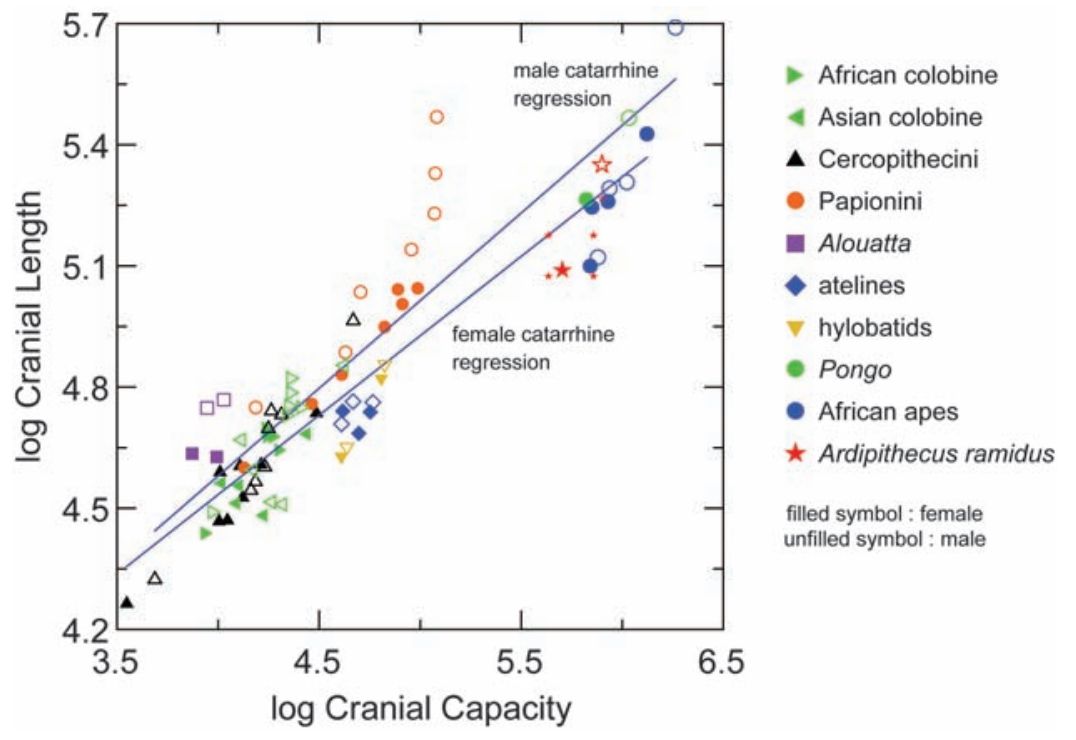

Fig. 5. Natural log-log plot of total cranial length against cranial capacity (24). Least-squares regression lines for the catarrhine subsets are fitted. African apes are Gorilla gorilla, $P$. troglodytes troglodytes, $P$. t. schweinfurth, and $P$. paniscus. Although $P$. $t$. schweinfurthi has a smaller body size, it has a larger ECC and cranial length than $P$. t. troglodytes. Bonobos have small skull size relative to ECC. Ar. ramidus (large red filled star) is plotted using the $A R A-V P-6 / 500$ body weight of $\sim 50 \mathrm{~kg} \mathrm{(23)} \mathrm{and} \mathrm{a}$ rough total cranial length estimate of $162.5 \mathrm{~mm}$ (fig. S7 and table S1). The boxed range of Ar. ramidus is depicted with a wide ECC range of 280 to $350 \mathrm{~cm}^{3}$. Possible skull length dispersion is depicted for a hypothetical situation in which $A R A-V P-6 / 500$ represents a small-skulled individual (within-sex correlation between body size and skull size is expected to be weak); most individuals may have had a larger skull size. The upper Ar. ramidus plots represent two SD positions using chimpanzee levels of variation as a model. The plotted Ar. ramidus range corresponds to approximately half of the species range of $P$. troglodytes, so its actual range of variation was greater. S. tchadensis (TM 266-01-60-1, large red unfilled star) is plotted from data in (13). See figs. S8 and S9 for further details. show that such facial features are highly variable within sexes and species. Hence, we do not consider its short face to be a species character of Ar. ramidus.

The $A r$ ramidus cranium: overall structure and comparisons. Digital restoration of the $A R A$ $V P-6 / 500$ cranium enables further observation and quantitative evaluation of its craniofacial architecture (24) (Figs. 2 and 4, fig. S7, and table $\mathrm{S} 1)$. The Ar. ramidus cranium shares enhanced relative upper facial projection (Fig. 4A) with Sahelanthropus (14) and later Pliocene hominids. However, the ape-like projecting midfacial muzzle of the Sahelanthropus/Ardipithecus face clearly differs from that of Australopithecus and early Homo, as shown metrically in Fig. 4B.

The Ardipithecus cranium also lacks the suite of derived masticatory features characteristic of later Australopithecus (2-5, 7-11, 43-47). We compared two such parameters, one of them explicitly examined in Sahelanthropus (14). Relative to biorbital breadth, maximum midfacial-zygomatic breadth at the orbital plane is considerably enhanced in Australopithecus (facial mask index, Fig. 4C) (10). Another measure, the overlap index (Fig. 4D), reflects the extent of anteroposterior overlap between the anterior-most limit of the origin of masseter and the postcanine tooth row $(10,43)$. In this measure, Ar. ramidus overlaps with Gorilla and the least derived end of the Australopithecus range.

Comparisons of Ar. ramidus and Australopithecus with the two extant Pan species reveal distinct cranial structures characteristic of each species. A pronounced feature of $P$. troglodytes is its elongate nasopharyngeal region and long anterior cranial base (see earlier in text). Associated morphological correlates include an anteroposteriorly elongate temporal fossa and infratemporal crest, as well as an anteriorly extended glenoid and preglenoid plane (48). The entire lower face/dentition is anteriorly displaced, an inference supported by morphological details such as the configuration of the posterior alveolar process. The P. troglodytes post- $\mathrm{M}^{3}$ maxillary tuberosity tends to be anteroposteriorly long, thereby adding to evidence for anterior displacement of the entire dental arcade relative to the pterygoid plates. The combined effect is an extremely prognathic lower face (Fig. 4E and fig. S6). We hypothesize that these craniofacial structures are highly derived but are not dietary adaptations; instead they are related to canine enlargement (34), perhaps in association with enhanced gape and/or increased aggression in P. troglodytes. Although most of these details cannot yet be directly observed in the Ar. ramidus cranium, it appears that such specializations were lacking. This is inferred from features such as the anteroposteriorly short glenoid and the $A R A-V P$ 6/500-based reconstruction with an anteroposteriorly short temporal fossa as in P. paniscus and G. gorilla.

The bonobo shares a long premaxilla and large incisors with the common chimpanzee, but 
lacks the extreme features of the latter. Instead, the bonobo cranium appears uniquely derived in its particularly small face and jaws (Fig. 4, F to H) (compared with both P. troglodytes and $A r$. ramidus). This structural pattern is consilient with the hypothesized size reduction of the entire bonobo dentition (34). The gorilla cranium is almost certainly derived in exhibiting an extreme anteriorly and inferiorly developed lower face [analytical results of $(13,14)]$. These and other facial and jaw features of the gorilla, some paralleling the Australopithecus condition (Fig. 4, $\mathrm{C}, \mathrm{G}$, and $\mathrm{H}$ ), are best interpreted as the combined effects of allometry (large absolute size) and functional adaptations to herbivory.

Cranial capacity, scaling, and cranial base flexion. Because the endocranial surface of the frontal pole region is preserved in $A R A-V P$ $6 / 500$, we were able to estimate cranial capacity from internal calvarial dimensions (length, breadth, and height). Multiple regressions based on modern African apes (total $n=18$, sexbalanced samples of $P$. troglodytes, $P$. pansicus and $G$. gorilla) yield an estimate of $300 \pm 10 \mathrm{~cm}^{3}$, with a larger range of 280 to $350 \mathrm{~cm}^{3}$ if we account for uncertainty that stems from combining $A R A-V P-6 / 500$ and the scaled $A R A-V P-1 / 500$ temporal/occipital portion (24). This small cranial capacity is comparable with that of female Pan (fig. S8).

Extending the Ar. ramidus reconstruction to include a rough approximation of total cranial length (fig. S7 and table S1) allows for a comparison of cranial size (maximum cranial length) with body size (fig. S9). Because subfamily level trends have been reported among catarrhines in relative endocranial volume (49), we also examined total skull length in relation to endocranial capacity (ECC). In addition to some colobines (in particular, Presbytis sensu stricto), atelines, hylobatids, and Pan, Ar. ramidus has the smallest relative cranial length among large-bodied anthropoids [as judged from regressions of cranial length on endocranial volume (Fig. 5)]. Because maximum cranial length controlled for endocranial volume must largely reflect facial and nuchal size, the results suggest a particularly gracile head in the $A R A-V P-6 / 500$ individual (50). At the same time, our scaling analysis shows that postcranially dimorphic species tend to exhibit a large cranial size relative to that of the endocranium, as well as a large degree of cranial size dimorphism. In this context, it is instructive that Ar. ramidus shares its relatively small cranial size with taxa that are weakly dimorphic both cranially and postcranially.

Despite its small cranial capacity, there is tantalizing evidence for advanced cranial base flexion in Ar. ramidus. This is seen from the steep orientation of its clivus, which directly reflects midsagittal flexion (figs. S10 and S11). However, because bonobos and Australopithecus overlap in measures of cranial base flexion $(33,41,51)$, it is uncertain whether $A r$ ramidus represents a primitive condition shared with bonobos or a more Australopithecus-like flexion involving the planum sphenoideum and/or greater orbital kyphosis (52).

The Sahelanthropus and Ardipithecus crania securely associate a relatively short basicranium with small cranial capacity. The hominid basicranial pattern and associated morphologies [such as foramen magnum orientation (24)] are widely held to be related to bipedality and upright posture $(12,13)$, despite a lack of empirical evidence to clearly support a functionally based correlation $(52,53)$. The Ar. ramidus cranium raises the alternative possibility that early hominid cranial base flexion was associated with neural reorganization that was already present in Sahelanthropus/Ardipithecus, as suggested for Pliocene Australopithecus (1, 54, 55). Such a hypothetical supposition is in part testable by both future fossil finds and by anticipated advances in our understanding of genomic expression patterns pertaining to brain function, structure, and morphogenesis.

Conclusions. Micro-CT-based evaluations of the Ar. ramidus cranial base confirm a derived basicranium of Ar. ramidus shared by both Sahelanthropus and Australopithecus. Our comparative analyses of $P$. troglodytes and $P$. paniscus suggest that this probably reflects basicranial organization unique to the hominid clade. The digitally reconstructed $\mathrm{Ar}$. ramidus skull further allows a variety of inferences about African ape and hominid evolution. Cranial capacity of preAustralopithecus hominids (as represented by Ar. ramidus and $S$. tchadensis) was probably slightly smaller than that of Australopithecus and also more comparable to Pan. The Ar. ramidus skull (and that of $S$. tchadensis) lacked the masticatory specializations of later Australopithecus, consistent with the dental evidence for an omnivore/frugivore niche lacking emphasis on hard and/or abrasive diets. Finally, comparisons of Ar. ramidus and extant African apes suggest that each is unique in aspects of its cranial anatomy. In particular, the common chimpanzee appears derived in its forwardly placed lower facial skeleton, possibly associated with increased aggression, whereas the bonobo is characterized by a secondary reduction of facial size. Ar. ramidus and Sahelanthropus lack these specialized morphologies of Pan and constitute the probable ancestral morphotype of Pliocene Australopithecus.

\section{References and Notes}

1. R. A. Dart, Nature 115, 195 (1925).

2. J. T. Robinson, Am. J. Phys. Anthropol. 12, 181 (1954).

3. P. V. T. Tobias, Olduvai Gorge Vol. 2, The Cranium and Maxillary Dentition of Australopithecus (Zinjanthropus) boisei (Cambridge Univ. Press, Cambridge, 1967).

4. B. Wood, N. Richmond, J. Anat. 197, 19 (2000).

5. T. D. White, in The Primate Fossil Record, W. C. Hartwig, Ed. (Cambridge Univ. Press, Cambridge, 2002), pp. 407-417.

6. Here we follow the taxonomy and phylogenetic scheme summarized in (5), which subsumes Paranthropus within the broadly defined genus Australopithecus. This is in part because monophyly of robust Australopithecus (Au. aethiopicus, Au, robustus, Au. boisei) is inconclusive, absent stratophenetic tests in South Africa $(5,56)$. We recognize seven species of Australopithecus spanning $\sim 4.2$ to $1.3 \mathrm{Ma}$ [the three "robust" species/chronospecies and $\mathrm{Au}$. anamensis, Au. afarensis, Au. africanus, and Au. garhi (most of the latter also considered

chronospecies)], as well as a single variable lineage of early Homo predating Homo erectus (56). We interpret a considerable amount of the observed metric and morphological variation to reflect temporal (phyletic evolutionary) and geographic differentiation (5). Others recognize more than 10 species in four or more genera of separate putative clades.

7. T. D. White, D. C. Johanson, W. H. Kimbel, S. Afr. J. Sci. 77, 445 (1981)

8. M. C. Dean, B. A. Wood, Am. J. Phys. Anthropol. 59, 157 (1982).

9. B. Wood, Koobi Fora Research Project Volume 4: Hominid Cranial Remains (Clarendon, Oxford, 1991).

10. W. H. Kimbel, Y. Rak, D. C. Johanson, Eds., The Skull of Australopithecus afarensis (Oxford Univ. Press, New York, 2004).

11. B. Asfaw et al., Science 284, 629 (1999).

12. M. Brunet et al., Nature 418, 145 (2002).

13. C. P. E. Zollikofer et al., Nature 434, 755 (2005).

14. F. Guy et al., Proc. Natl. Acad. Sci. U.S.A. 102, 18836 (2005).

15. B. Wood, Nature 418, 133 (2002).

16. B. Senut, M. Pickford, C. R. Palevol 3, 265 (2004).

17. M. H. Wolpoff, ]. Hawks, B. Senut, M. Pickford, ]. Ahern, Paleoanthropology 2006, 36 (2006).

18. T. D. White et al., Science 326, 64 (2009).

19. T. D. White, G. Suwa, B. Asfaw, Nature 371, 306 (1994).

20. C. O. Lovejoy et al., Science 326, 70 (2009)

21. C. O. Lovejoy et al., Science 326, 71 (2009).

22. C. O. Lovejoy et al., Science 326, 72 (2009).

23. C. O. Lovejoy et al., Science 326, 73 (2009).

24. Materials and methods are available as supporting material on Science Online.

25. Adding the scaled $A R A-V P-1 / 500$ temporal/occipital portion to the $A R A-V P-6 / 500$ cranial reconstruction is justified by general similarities in the major parts preserved in both specimens (basioccipital, glenoid, and zygomatic root areas) (24).

26. M. S. Schaefer, Am. J. Phys. Anthropol. 110, 467 (1999).

27. J. C. M. Ahern, Am. J. Phys. Anthropol. 127, 267 (2005)

28. We initially reported that, in Ar. ramidus, the bicarotid chord intersects the basion (19). Subsequently, the Sahelanthropus cranium was similarly described as having a basion intersected by the bicarotid chord and "touched" by the biporion line (12). The latter depends on cranial orientation, which was not specified in that publication. Other workers measured the basion-tobiporion (or basion-to-bicarotid) distance, either by projection in standard (Frankfurt horizontal) orientation (27) or by direct measurement in basal view (26). Differences between and within methods are potentially large when biporion or bitympanic chords are used, due to the vertical offset of these landmarks from the basion. An additional difficulty arises in evaluating fragmentary fossils that are rarely complete enough to apply methods relying on standard orientations. We therefore opted to measure the basion-to-bicarotid distance in locally determined basilar orientation (24)

29. S. A. Luboga, B. A. Wood, Am. J. Phys. Anthropol. 81, 67 (1990).

30. Earlier studies of Australopithecus external basicranial shape (8) combined radiographic and direct caliper measures, apparently without due regard to the potential effects of orientation in such metric evaluations. These results suggested an extreme anterior position of the basion in robust Australopithecus crania, but not in A. africanus. However, this dichotomy was not replicated in a more recent study (27) that examined the basion-tobicarotid chord distance in Frankfurt horizontal projection. Our own results also failed to reveal clear differences in basion position between $\mathrm{Au}$. africanus and eastern African robust Australopithecus (fig. S2). Rather, all Australopithecus taxa appear broadly comparable in their positions of the basion, which is consistently located slightly posterior to the carotid foramina. 
31. We agree with previous workers $(26,27)$ that, relative to the basion, the carotid foramen is more anteriorly situated in modern humans than in Plio-Pleistocene hominids. Thus, considerable overlap in range of variation occurs between chimpanzees and humans in measures such as basion-to-biporion or basion-to-bicarotid distances $(26,27)$. However, modern human and chimpanzee cranial bases differ considerably in overall structure, and the importance of isolated metrics must be considered carefully. We observe that jugular and carotid sizes are much larger in humans than in apes or MioPliocene hominids and that this directly affects measures of basion position. Larger jugular and carotid size almost certainly stems from allometric enlargement of the cranial vascular system in extremely encephalized Homo. Thus, overlap in parameters such as the basion-to-bicarotid chord between humans and chimpanzees has little consequence in evaluating and interpreting Ar. ramidus.

32. Following Guy et al. (14), we term the pre- and postchordal portions of the cranial base the anterior and posterior cranial base, respectively.

33. R. C. McCarthy, J. Hum. Evol. 40, 41 (2001).

34. G. Suwa et al., Science 326, 69 (2009).

35. C. V. Ward, M. G. Leakey, A. Walker, J. Hum. Evol. 41, 255 (2001).

36. W. H. Kimbel et al., J. Hum. Evol. 51, 134 (2006).

37. T. D. White, G. Suwa, S. Simpson, B. Asfaw, Am. J. Phys. Anthropol. 111, 45 (2000).

38. M. Brunet et al., Nature 434, 752 (2005).

39. Y. Haile-Selassie, G. Suwa, T. D. White, in Ardipithecus kadabba: Late Miocene Evidence from the Middle Awash Valley, Y. Haile-Selassie, G. WoldeGabriel, Eds. (Univ. of California Press, Berkeley, CA, 2009), pp. 159-236.

40. Equivalent measures of mid-torus thickness were taken on a random subset of the $P$. troglodytes sample of the cranial base analysis (24) with the following results: males ( $n=5)$, mean $8.4 \mathrm{~mm}$, range 6.4 to $12.1 \mathrm{~mm}$; females $(n=6)$, mean $6.5 \mathrm{~mm}$, range 5.0 to $8.2 \mathrm{~mm}$. Guy et al. (14) report size-standardized values of midtorus thickness of the $S$. tchadensis cranium. This can be converted to an absolute mid-torus thickness of $13.8 \mathrm{~mm}$

41. R. Fenart, R. Deblock, Pan paniscus et Pan troglodytes Craniométrie. Ann. Mus. Roy. Afrique Centr. Ser. IN-8: no. 204 (Royal Museum for Central Africa, Tervuren, Belgium, 1973).

42. J. M. Plavcan, J. Hum. Evol. 42, 579 (2002).

43. Y. Rak, The Australopithecine Face (Academic Press, New York, 1983).

44. W. H. Kimbel, T. D. White, D. C. Johanson, Am. J. Phys. Anthropol. 64, 337 (1984).

45. A. Walker, R. E. Leakey, J. M. Harris, F. H. Brown, Nature 322, 517 (1986).

46. G. Suwa et al., Nature 389, 489 (1997).

47. C. A. Lockwood, P. V. Tobias, J. Hum. Evol. 36, 637 (1999).

48. C. A. Lockwood, J. M. Lynch, W. H. Kimbel, J. Anat. 201, 447 (2002)

49. K. Isler et al., J. Hum. Evol. 55, 967 (2008).

50. Both small ECC and small relative cranial length provide additional support to our inference that $A R A-V P-6 / 500$ is a female $(18,34)$.

51. F. Spoor, S. Afr. J. Sci. 93, 182 (1997).

52. C. Ross, M. Henneberg, Am. J. Phys. Anthropol. 98, 575 (1995).

53. ]. Biegert, in Classification and Human Evolution, S. L. Washburn, Ed. (Aldine, Chicago, 1963), pp. 116-145.

54. R. L. Holloway, Nature 303, 420 (1983).

55. R. L. Holloway, R. J. Clarke, P. V. Tobias, C. R. Palevol 3, 287 (2004)

56. G. Suwa, T. D. White, F. C. Howell, Am. J. Phys. Anthropol. 101, 247 (1996).

57. G. Suwa, J. Anthrop. Soc. Nippon 89, 303 (1981).

58. M. Caix, G. Outrequin, Anat. Clin. 1, 259 (1979).
59. F. Spoor et al., Proc. Natl. Acad. Sci. U.S.A. 104, 10808 (2007)

60. F. Spoor, F. Zonneveld, Yearb. Phys. Anthropol. 41, 211 (1998).

61. B. Brown, A. Walker, C. V. Ward, R. E. Leakey, Am. J. Phys. Anthropol. 91, 137 (1993).

62. We thank NSF (this material is based on work supported by grants SBR-82-10897, 93-18698, 95-12534, 9632389, 99-10344, and 03-21893 HOMINID-RHOI) and the Japan Society for the Promotion of Science (grant 11691176, 16405016, 17207017, and 21255005) for funding; the Ministry of Tourism and Culture, the Authority for Research and Conservation of the Cultural Heritage, and the National Museum of Ethiopia for permissions and facilitation; the Afar Regional Government, the Afar people of the Middle Awash, and many other field workers for contributing directly to the data; $\mathrm{H}$. Gilbert for graphics assistance for Figs. 1 and 2; and the following institutions and staff for access to comparative materials: National Museum of Ethiopia, National Museums of Kenya, Transvaal Museum South Africa, Cleveland Museum of Natural History, Royal Museum for Central Africa Tervuren, Naturalis Leiden, the University of California at Berkeley Human Evolution Research Center, and the Department of Zoology of the National Museum of Nature and Science Tokyo. We also thank M. Brunet, F. Guy, M. Plavcan, M. Ponce de León, and C. Zollikofer for cooperation with comparative data.

\section{Supporting Online Material}

www.sciencemag.org/cgi/content/full/326/5949/68/DC1

Materials and Methods

Figs. S1 to S11

Table S1

References

4 May 2009; accepted 31 August 2009

10.1126/science. 1175825 